\title{
SECOND ORDER OPTIMALITY CONDITIONS FOR MATHEMATICAL PROGRAMMING WITH SET FUNCTIONS
}

\author{
J. H. CHOU, WEI-SHEN HSIA AND TAN-YU LEE ${ }^{1}$
}

(Received 24 May 1983; revised 5 March 1984)

\begin{abstract}
Second order necessary and sufficient conditions are given for a class of optimization problems involving optimal selection of a measurable subset from a given measure subspace subject to set function inequalities. Relations between twice-differentiability at $\Omega$ and local convexity at $\Omega$ are also discussed.
\end{abstract}

\section{Introduction}

Let $(X, \mathscr{A}, \mu)$ be a finite measure space and $F, G_{i}, \ldots, G_{m}$ be real-valued set functions on $\mathscr{A}$. The problem considered in this paper is to find a measurable set $\Omega^{*} \in \mathscr{A}$ which minimizes $F(\Omega)$ subject to constraints $G_{i}(\Omega) \leqslant 0, i=1, \ldots, m$. This type of optimization problem has received attention lately due to its diverse applications and theoretical interest. These include applications in fluid flow [1], electrical insulator design [3], optimal plasma confinement [12], first order necessary and sufficient optimal conditions [11], and duality theories set functions [6] and [7].

The difficulty of the above problem, as pointed out by Morris in [11], lies in the poorly structured feasible domain which is not convex, not open, and actually nowhere dense. Morris [11] overcame these difficulties and derived several necessary and sufficient optimality conditions with properly defined notions of firstorder differentiability and convexity of set functions. By continuing to work in the setting of Morris [11] and Luenberger [8], Lai, Yang and Hwang [7] proved the Fenchel duality theorem for set functions.

\footnotetext{
${ }^{1}$ Department of Mathematics, The University of Alabama, University, Alabama 35486, U.S A.

(C) Copyright Australian Mathematical Society 1985, Serial-fee code 0334-2700/85
} 
The purpose of this paper is to obtain second-order necessary and sufficient optimality conditions for the above problem. In Section 2, we begin with a definition of second differentiability of a set function on a measure space. This is followed by several theorems concerning properties of a set function with second differentiability. Section 3 contains the main results of this paper, in which sufficient conditions are presented in a way close to that in [4], [10].

Numerous results on necessary and sufficient optimality conditions in optimization problems for point functions under second order differentiability assumptions have been given by many researchers; some recent ones include [2] and [5].

\section{Second differentiability of set functions}

Throughout this paper, we assume that the measure space $(X, \mathscr{A}, \mu)$ is finite and atomless with $L_{1}(X, \mathscr{A}, \mu)=L_{1}(\mu)$ separable. Let $\rho$ be a pseudometric on $\mathscr{A}$ defined by $\rho\left(\Omega_{1}, \Omega_{2}\right)=\mu\left(\Omega_{1} \Delta \Omega_{2}\right)$ for $\Omega_{1}, \Omega_{2} \in \mathscr{A}$, and we identify any set $\Omega \in \mathscr{A}$ with its characteristic function $\chi_{\Omega} \in L_{1}(\mu)$. Thus $\mathscr{A}$ can be regarded as a subset $\chi_{\mathscr{\infty}}=\left\{\chi_{\Omega} \mid \Omega \in \mathscr{A}\right\}$ of $L_{1}(\mu)$. Note that $\rho\left(\Omega_{1}, \Omega_{2}\right)=\left\|\chi_{\Omega_{1}}-\chi_{\Omega_{2}}\right\|_{L_{1}}$. For $f \in L_{1}(\mu)$ and $w \in L_{1}\left(\mu_{1} \times \mu_{1}\right)$ we denote the integral $\int_{\Omega} f$ by the functional notation $\left\langle f, \chi_{\Omega}\right\rangle, \int_{\Omega_{1} \times \Omega_{2}} w$ by $\left\langle w, \chi_{\Omega_{1}} \times \chi_{\Omega_{2}}\right\rangle$. The diagonal of $w$, denoted by diag $w$, is defined as a function on $\mathscr{A}$ in the following way:

$$
\operatorname{diag} w(\Omega)=\left\langle w, \chi_{\Omega} \times \chi_{\Omega}\right\rangle, \quad \Omega \in \mathscr{A} .
$$

Moreover, $\operatorname{diag} w$ is said to be $w^{*}$-continuous if $\chi_{\Omega_{n}} \rightarrow^{w^{*}} \chi_{\Omega}$ implies that $\operatorname{diag} w\left(\Omega_{n}\right) \rightarrow \operatorname{diag} w(\Omega)$ where $\chi_{\Omega_{n}} \rightarrow{ }^{*} \chi_{\Omega}$ means $\left\langle f, \chi_{\Omega_{n}}\right\rangle \rightarrow\left\langle f, \chi_{\Omega}\right\rangle$ for all $f \in L_{1}(\mu)$.

Definition 1 [11]. A set function $F: \mathscr{A} \rightarrow R$ is said to be differentiable at $\Omega_{0} \in \mathscr{A}$ if there exists $D F_{\Omega_{0}} \in L_{1}(\mu)$, called the first derivative of $F$ at $\Omega_{0}$, such that

$$
F(\Omega)=F\left(\Omega_{0}\right)+\left\langle D F_{\Omega_{0}}, \chi_{\Omega}-\chi_{\Omega_{0}}\right\rangle+E\left(\Omega_{0}, \Omega\right) .
$$

where $E\left(\Omega_{0}, \Omega\right)=o\left[\rho\left(\Omega_{0}, \Omega\right)\right]$, i.e., $\lim _{\rho\left(\Omega_{0}, \Omega\right) \rightarrow 0}\left[E\left(\Omega_{0}, \Omega\right) / \rho\left(\Omega_{0}, \Omega\right)\right]=0$.

REMARKs. Definition 1 differs from that of the usual Fréchet derivative [8] in that $\Omega \rightarrow \Omega_{0}$ passing through only points of the subset $\chi_{\mathscr{A}}$ which is not even a linear subspace. However, if $\tilde{F}$ is a Fréchet differentiable functional on $L_{1}(\mu)$ and if we define a set function $F$ on $\mathscr{A}$ by $F(\Omega)=\tilde{F}\left(\chi_{\Omega}\right)$, then $F$ is a differentiable set function. In this case, note that the Fréchet derivative of $\tilde{F}$ at $\Omega$ coincides with $D F_{\Omega}$ due to the uniqueness of the derivative of a set function [11].

LEMMA 1 [11]. For any $\Omega \in \mathscr{A}$ and $\alpha \in[0,1]$ there exists a sequence $\left\{\Omega_{n}\right\}$ with $\Omega_{n} \subset \Omega$ for all $n$ and $\chi_{\Omega_{n}} \rightarrow{ }^{* *} \alpha \chi_{\Omega}$. 
LeMma 2. For $\Omega, \Omega_{0} \in \mathscr{A}$ and $\alpha \in[0,1]$ there exists a sequence $\left\{\Omega_{n}(\alpha)\right\}$ in $\mathscr{A}$ such that $\chi_{\Omega_{n}(\alpha)}-\chi_{\Omega_{0}} \rightarrow{ }^{* *} \alpha\left(\chi_{\Omega}-\chi_{\Omega_{0}}\right)$.

Proof. Let $\Omega^{+}=\Omega \sim \Omega_{0}$ and $\Omega^{-}=\Omega_{0} \sim \Omega$. Then

$$
\chi_{\Omega}-\chi_{\mathbf{\Omega}_{0}}=\chi_{\mathbf{\Omega}^{+}}-\chi_{\Omega^{-}}
$$

By Lemma 1 there exist sequences $\left\{\Omega_{n}^{ \pm}(\alpha)\right\}$ satisfying

$$
\chi_{\Omega_{n}^{ \pm}(\alpha)} \stackrel{w^{*}}{\rightarrow} \alpha \chi_{\Omega^{ \pm}}
$$

Let $\Omega_{n}(\alpha)$ denote $\left(\Omega_{n}^{+}(\alpha) \cup \Omega_{0}\right) \sim \Omega_{n}^{-}(\alpha)$, then

$$
\chi_{\Omega_{n}(\alpha)}-\chi_{\Omega_{0}}=\chi_{\Omega_{n}^{+}(\alpha)}-\chi_{\Omega_{n}^{-}(\alpha)}
$$

and

$$
\chi_{\Omega_{n}(\alpha)}-\chi s_{\Omega_{0}} \stackrel{w^{*}}{\rightarrow} \alpha\left(\chi_{\Omega^{+}}-\chi_{\Omega^{-}}\right)=\alpha\left(\chi_{\Omega}-\chi_{\Omega_{0}}\right) . \quad \text { Q.E.D. }
$$

Definition 2. A set function $F: \mathscr{A} \rightarrow R$ is said to be twice differentiable at $\Omega_{0} \in \mathscr{A}$ if it has a first derivative $D F_{\Omega_{0}}$ at $\Omega_{0}$, and there exists $D^{2} F_{\Omega_{0}} \in L_{1}(\mu \times \mu)$ such that the function defined by $q_{\Omega_{0}}(\Omega)=\operatorname{diag} D^{2} F_{\Omega_{0}}\left(\chi_{\Omega}-\chi_{\Omega_{0}}\right)$, called the second derivative of $F$ at $\Omega_{0}$, is $w^{*}$-continuous, is $o\left[\rho\left(\Omega, \Omega_{0}\right)\right]$, and satisfies

$$
F(\Omega)=F\left(\Omega_{0}\right)+\left\langle D F_{\Omega_{0}}, \chi_{\Omega}-\chi_{\Omega_{0}}\right\rangle+\left\langle D^{2} F_{\Omega_{0}},\left(\chi_{\Omega}-\chi_{\Omega_{0}}\right)^{2}\right\rangle+E\left(\Omega, \Omega_{0}\right)
$$

where $E\left(\Omega, \Omega_{0}\right)=o\left[\rho^{2}\left(\Omega, \Omega_{0}\right)\right]$, i.e. $\lim _{\rho\left(\Omega, \Omega_{0}\right) \rightarrow 0}\left[E\left(\Omega, \Omega_{0}\right) / \rho^{2}\left(\Omega, \Omega_{0}\right)\right]=0$.

Lemma 3. If $F$ is twice differentiable at $\Omega_{0}$, then for any $\Omega \in \mathscr{A}$ and $\alpha \in[0,1]$ there exists a sequence $\left\{\Omega_{n}(\alpha)\right\}$ in $\mathscr{A}$ such that

$$
\chi_{\Omega_{n}(\alpha)}-\chi_{\Omega_{0}} \stackrel{\omega^{*}}{\rightarrow} \alpha\left(\chi_{\Omega}-\chi_{\Omega_{0}}\right)
$$

and

$$
\begin{aligned}
\lim _{n \rightarrow \infty} F\left(\Omega_{n}(\alpha)\right)= & F\left(\Omega_{0}\right)+\alpha\left\langle D F_{\Omega_{0}}, \chi_{\Omega}-\chi_{\Omega_{0}}\right\rangle \\
& +\alpha^{2}\left\langle D^{2} F_{\Omega_{0}},\left(\chi_{\Omega}-\chi_{\Omega_{0}}\right)^{2}\right\rangle+o\left(\alpha^{2}\right)
\end{aligned}
$$

Proof. Fix $\Omega$. For $\alpha \in[0,1]$, let $\left\{\Omega_{n}(\alpha)\right\}$ be the sequence in Lemma 2 satisfying

$$
\chi_{\Omega_{n}(\alpha)}-\chi_{\Omega_{0}} \stackrel{\omega^{*}}{\rightarrow} \alpha\left(\chi_{\Omega}-\chi_{\Omega_{0}}\right)
$$

Then

$$
\begin{aligned}
\lim _{n \rightarrow \infty} F\left(\Omega_{n}(\alpha)\right)= & F\left(\Omega_{0}\right)+\alpha\left\langle D F_{\Omega_{0}}, \chi_{\Omega}-\chi_{\Omega_{0}}\right\rangle \\
& +\alpha^{2}\left\langle D^{2} F_{\Omega_{0}},\left(\chi_{\Omega}-\chi_{\Omega_{0}}\right)^{2}\right\rangle+\lim _{n \rightarrow \infty} E\left(\Omega_{n}(\alpha), \Omega_{0}\right) .
\end{aligned}
$$

We need only to show that $\lim _{n \rightarrow \infty} E\left(\Omega_{n}(\alpha), \Omega_{0}\right)=o\left(\alpha^{2}\right)$. 
It suffices to show that, given $\varepsilon>0$, there exists $\delta>0$ such that $0 \leqslant \alpha<\delta$ implies $\lim _{n \rightarrow \infty}\left|E\left(\Omega_{n}(\alpha), \Omega_{0}\right)\right| \leqslant \varepsilon \alpha^{2}$. Since $E\left(\Omega^{\prime}, \Omega_{0}\right)=o\left[\rho^{2}\left(\Omega^{\prime}, \Omega_{0}\right)\right]$ there exists $\gamma>0$ such that $\left|E\left(\Omega^{\prime}, \Omega_{0}\right)\right| \leqslant \varepsilon \rho^{2}\left(\Omega^{\prime}, \Omega_{0}\right)$ for $\Omega^{\prime} \in \mathscr{A}$ satisfying $\rho\left(\Omega^{\prime}, \Omega_{0}\right)<\gamma$. Now let $\delta=\gamma / \rho\left(\Omega, \Omega_{0}\right)$. Then

$$
\rho\left(\Omega_{n}(\alpha), \Omega_{0}\right)=\left\|\chi_{\Omega_{n}(\alpha)}-\chi_{\Omega_{0}}\right\|_{L_{1}} \rightarrow \alpha\left\|\chi_{\Omega}-\chi_{\Omega_{0}}\right\|_{L_{1}}=\alpha \rho\left(\Omega, \Omega_{0}\right)
$$

implies that for $\alpha<\delta$ and for all large $n$ 's we have $\rho\left(\Omega_{n}(\alpha), \Omega_{0}\right)<\gamma$. Hence $\left|E\left(\Omega_{n}(\alpha), \Omega_{0}\right)\right| \leqslant \varepsilon \rho^{2}\left(\Omega_{n}(\alpha), \Omega_{0}\right)$ and therefore $\lim _{n \rightarrow \infty} E\left(\Omega_{n}(\alpha), \Omega_{0}\right) \leqslant \varepsilon \alpha^{2}$. Q.E.D.

THEOREM 1. If $F$ is twice differentiable at $\Omega_{0}$, then both the first and second derivative are unique.

Proof. Let $f$ and $\bar{f}$ both be the first derivatives of $F$ at $\Omega_{0}$; and $h$ and $\bar{h}$ the second derivatives. Set $g=f-\bar{f}$ and $\gamma=h-\bar{h}$. Then $\left\langle g, \chi_{\Omega}-\chi_{\Omega_{0}}\right\rangle=$ $o\left[\rho\left(\Omega, \Omega_{0}\right)\right]$ and $\gamma(\Omega)=o\left[\rho^{2}\left(\Omega, \Omega_{0}\right)\right]$ where $\gamma(\Omega)=\left\langle w,\left(\chi_{\Omega}-\chi_{\Omega_{0}}\right)^{2}\right\rangle$ for some $w \in L_{1}(\mu \times \mu)$. Given $\Omega \in \mathscr{A}$, by Lemma 2 , for any $\alpha \in[0,1]$ there exists a sequence $\left\{\Omega_{n}(\alpha)\right\}$ with

$$
\chi_{\Omega_{n}(\alpha)}-\chi_{\Omega_{0}} \stackrel{w^{*}}{\rightarrow} \alpha\left(\chi_{\Omega}-\chi_{\Omega_{0}}\right)
$$

Then

$$
\left\langle g, \chi_{\Omega_{n}(\alpha)}-\chi_{\Omega_{0}}\right\rangle \rightarrow \alpha\left\langle g, \chi_{\Omega}-\chi_{\Omega_{0}}\right\rangle
$$

and

$$
\left\langle w,\left(\chi_{\Omega_{n}(\alpha)}-\chi_{\Omega_{0}}\right)^{2}\right\rangle \rightarrow \alpha^{2}\left\langle w,\left(\chi_{\Omega}-\chi_{\Omega_{0}}\right)^{2}\right\rangle .
$$

Since $\rho\left(\Omega_{n}(\alpha), \Omega_{0}\right) \rightarrow \alpha \rho\left(\Omega, \Omega_{0}\right)$, by a similar argument as used in the proof of Lemma 3 we have

$$
\lim _{n \rightarrow \infty}\left\langle g, \chi_{\Omega_{n}(\alpha)}-\chi_{\Omega_{0}}\right\rangle=\alpha\left\langle g, \chi_{\Omega}-\chi_{\Omega_{0}}\right\rangle=o(\alpha)
$$

and

$$
\alpha^{2}\left\langle w,\left(\chi_{\Omega}-\chi_{\Omega_{0}}\right)^{2}\right\rangle=o\left(\alpha^{2}\right) .
$$

This implies that $\left\langle g, \chi_{\Omega}-\chi_{\Omega_{0}}\right\rangle=0$ and $\left\langle w,\left(\chi_{\Omega}-\chi_{\Omega_{0}}\right)^{2}\right\rangle=0$ for any $\Omega \in \mathscr{A}$. Let $\Omega_{+}=g^{-1}([0, \infty)]$ and $\Omega_{-}=g^{-1}((-\infty, 0])$. Then $\left\langle g, \chi_{\Omega_{0}}\right\rangle=\left\langle g, \chi_{\Omega_{+}}\right\rangle \geqslant 0$ and $\left\langle g, \chi_{\Omega_{0}}\right\rangle=\left\langle g, \chi_{\Omega_{-}}\right\rangle \leqslant 0$ which implies $\left\langle g, \chi_{\Omega_{0}}\right\rangle=0$. Therefore, $\left\langle g, \chi_{\Omega}\right\rangle=$ 0 for all $\Omega \in \mathscr{A}$. Hence, $g=0$, a.e. on $X$. Similarly, $\gamma=0$ a.e. on $X$. Q.E.D.

Remarks. (i) If $F$ is twice differentiable at $\Omega_{0}$, then $F$ is differentiable at $\Omega_{0}$. Since $q_{\Omega_{0}}(\Omega) \in o\left[\rho\left(\Omega, \Omega_{0}\right)\right]$ by assumption, hence $D F_{\Omega_{0}}$ is unique by Proposition 
2.2 [10]. The first derivative of $F$ is a linear functional on $\mathscr{A}$ defined by $\Omega \rightarrow\left\langle D F_{\Omega_{0}}, \chi_{\Omega}-\chi_{\Omega_{0}}\right\rangle$ rather than just $D F_{\Omega_{0}}$, an $L_{1}$-function. However, we may identify the first derivative with $D F_{\Omega_{0}}$ [10]. For the second derivative $q_{\Omega_{0}}(\Omega)=$ $\left\langle D^{2} F_{\Omega_{0}},\left(\chi_{\Omega}-\chi_{\Omega_{0}}\right)_{1}^{2}\right\rangle$, it is the quadratic form defined by $D^{2} F_{\Omega_{0}}$. We may always assume $D^{2} F_{\Omega_{0}}$ is symmetric in Definition 2, i.e., $D^{2} F_{\Omega_{0}}(x, y)=D^{2} F_{\Omega_{0}}(y, x), \forall x, y$ $\in \mathscr{A}$, since $\frac{1}{2}\left(D^{2} F_{\Omega_{0}}(x, y)+D^{2} F_{\Omega_{v}}(y, x)\right)$ is symmetric and defines the same quadratic form.

(ii) If $F$ is countably additive and absolutely continuous with respect to $\mu$, then $D F_{\Omega}$ is simply the Radon-Nikodym derivative $D f / d \mu$, and the second derivative $q_{\Omega} \equiv 0$ for all $\Omega \in \mathscr{A}$.

(iii) Another example of a twice differentiable set function is $F(\Omega)=$ $h\left(\int_{\Omega} v_{1} d \mu, \ldots, \int_{\Omega} v_{n} d \mu\right)$ where $h: R^{n} \rightarrow R$ is differentiable and $v_{1}, \ldots, v_{n}$ are in $L_{1}(\mu)$. Then its first derivative

$$
D F_{\Omega}=\sum_{i=1}^{n} h_{i}\left(\int_{\Omega} v_{1} d_{\mu}, \ldots, \int_{\Omega} v_{n} d_{\mu}\right) v_{i}
$$

and its second derivative

$$
D^{2} F_{\Omega}=\sum_{j=1}^{n} \sum_{i=1}^{n} h_{t j}\left(\int_{\Omega} v_{1} d_{\mu}, \ldots, \int_{\Omega} v_{n} d_{\mu}\right) v_{i} v_{j}
$$

where $h_{i}$ denotes the $i$ th first partial derivative, and $h_{i j}$ is the $i j$ th second partial derivative of $h$.

(iv) If $F$ and $G$ are differentiable (twice differentiable) at $\Omega_{0}$, then for $c \in R$, $c \cdot F$, and $F \pm G$ are differentiable (twice differentiable) at $\Omega_{0}$.

In order to obtain sufficient conditions for a constrained local minimum, Morris [11] introduced the concept of local convexity of a set function as follows.

Definition 3 [11]. A differentiable set function $F: \mathscr{A} \rightarrow R$ is locally convex at $\Omega_{0}$ if there exists $\varepsilon>0$ such that $\rho\left(\Omega_{0}, \Omega\right)<\varepsilon$ implies

$$
F(\Omega) \geqslant F\left(\Omega_{0}\right)+\left\langle D F_{\Omega_{0}}, \chi_{\Omega}-\chi_{\Omega_{0}}\right\rangle .
$$

The following lemmas give relationships between local convexity of a set function and its second derivative.

LEMMA 4. Let $F: \mathscr{A} \rightarrow R$ be a set function which is twice differentiable at $\Omega_{0}$. If $F$ is locally convex at $\Omega_{0}$ then there exists $\varepsilon>0$ such that $\rho\left(\Omega_{0}, \Omega\right)<\varepsilon$ implies $\left\langle D^{2} F_{\Omega_{0}},\left(\chi_{\Omega}-\chi_{\Omega_{0}}\right)^{2}\right\rangle \geqslant 0$, i.e., $D^{2} F_{\Omega_{0}}$ is locally positive semidefinite.

Proof. Using the sequence $\left\{\Omega_{n}(\alpha)\right\}$ given in Lemma 3, the proof of this lemma is similar to that of Theorem 1 in $[9$, page 89]. Q.E.D. 
LEMMA 5. Let $F: \mathscr{A} \rightarrow R$ be a set function which is twice differentiable at $\Omega_{0}$. If there exists $\gamma>0$ such that

$$
\left\langle D^{2} F_{\Omega_{0}},\left(\chi_{\Omega}-\chi_{\Omega_{0}}\right)^{2}\right\rangle \geqslant \gamma \rho^{2}\left(\Omega, \Omega_{0}\right)
$$

for all $\Omega$ with $\rho\left(\Omega, \Omega_{0}\right)<\varepsilon$ for some $\varepsilon>0$, then $F$ is locally convex at $\Omega_{0}$.

Proof. The result follows directly from Lemma 3 and the definition of " $o$ ". Q.E.D.

\section{Optimality conditions of second order}

In this section we consider the problem mentioned at the beginning of Section 1:

$$
\operatorname{Min} F(\Omega) \quad \text { subject to } G_{t}(\Omega) \leqslant 0, i=1, \ldots, m \text {. }
$$

$\Omega_{0} \in \mathscr{A}$ is a local minimum for problem (1) if there exists $\varepsilon>0$ such that for $\Omega$ satisfying $\rho\left(\Omega_{0}, \Omega\right)<\varepsilon, G_{i}(\Omega) \leqslant 0, i=1, \ldots, m$, it follows that $F(\Omega) \geqslant F\left(\Omega_{0}\right)$.

The first-order necessary condition to this problem was given by Morris in [11].

\section{THEOREM 2 [11]. Suppose}

(i) $F, G_{1}, \ldots, G_{m}$ are differentiable at $\Omega^{*}$ with first derivatives $D F_{\Omega^{*}}, D G_{\Omega^{*}}^{1}, \ldots, D G_{\Omega^{*}}^{m}$, respectively.

(ii) $\Omega^{*}$ is a local minimum of problem (1), and

(iii) $\Omega^{*}$ is regular, i.e., there exists a set $\Omega_{1} \in \mathscr{A}$ with $G_{1}\left(\Omega^{*}\right)+\left\langle D G_{\Omega^{*}}^{\prime}, \chi_{\Omega_{1}}-\right.$ $\left.\chi_{\Omega^{*}}\right\rangle\left\langle 0, i=1, \ldots, m\right.$. Then there exists nonnegative reals $\lambda_{1}, \ldots, \lambda_{m}$ such that

$$
\left\{\begin{array}{l}
\left\langle D F_{\Omega^{*}}+\sum_{i=1}^{m} \lambda_{i} D G_{\Omega^{*}}^{i}, \chi_{\Omega}-\chi_{\Omega^{*}}\right\rangle \geqslant 0 \text { for all } \Omega \in \mathscr{A}, \text { and } \\
\lambda_{i}=0 \quad \text { if } G_{i}\left(\Omega^{*}\right)<0 .
\end{array}\right.
$$

A set of nonnegative reals $\lambda_{1}, \ldots, \lambda_{m}$ for which (2) holds is called a Lagrangian multiplier for problem (1) at $\Omega^{*}$ and the associated Lagrangian function is defined as $L(\Omega)=F(\Omega)+\sum_{l-1}^{m} \lambda_{l} G_{i}(\Omega)$. We denote the feasible region of problem (1) by $S=\left\{\Omega \in \mathscr{A} \mid G_{i}(\Omega) \leqslant 0, i=1, \ldots, m\right\}$, the index set of active constraints at $\Omega^{*}$ by $I\left(\Omega^{*}\right)=\left\{i \mid G_{i}\left(\Omega^{*}\right)=0\right\}$, and the first derivative of $L$ at $\Omega$ by $D L_{\Omega}=D F_{\Omega}+$ $\sum_{i=1}^{m} \lambda_{i} D G_{\Omega}^{\prime}$.

THeOREM 3 (Second-Order Necessary Condition). Let $F, G_{1}, \ldots, G_{m}$ be twice differentiable at $\Omega^{*}$. Suppose $\Omega^{*}$ is a local minimum of problem (1) and suppose 
$L(\Omega)=F(\Omega)+\sum_{i-1}^{m} \lambda_{i} G_{i}(\Omega)$ is a Lagrangian function associated with a set of Lagrangian multipliers $\lambda_{1}, \ldots, \lambda_{m}$ for problem (1) at $\Omega^{*}$. Then

$$
\left\langle D^{2} L_{\Omega^{*}},\left(\chi_{\Omega}-\chi_{\Omega^{*}}\right)^{2}\right\rangle \geqslant 0
$$

for all $\Omega \in S$ satisfying

$$
\begin{aligned}
& \left\langle D L_{\Omega^{*}}, \chi_{\Omega}-\chi_{\Omega^{*}}\right\rangle=0, \\
& \left\langle D G_{\Omega^{*}}^{i}, \chi_{\Omega}-\chi_{\Omega^{*}}\right\rangle<0, \quad i \in I\left(\Omega^{*}\right),
\end{aligned}
$$

and $\lambda_{i} G_{i}(\Omega)=0, i=1, \ldots, m$.

Proof. For any $\Omega \in S$ satisfying (3) we have $F(\Omega)=L(\Omega)$ and it follows that

$$
\begin{aligned}
F(\Omega)-F\left(\Omega^{*}\right) & =L(\Omega)-L\left(\Omega^{*}\right) \\
& =\left\langle D^{2} L_{\Omega^{*}},\left(\chi_{\Omega}-\chi_{\Omega^{*}}\right)^{2}\right\rangle+E\left(\Omega, \Omega^{*}\right)
\end{aligned}
$$

where $E\left(\Omega, \Omega^{*}\right)=o\left[\rho^{2}\left(\Omega, \Omega^{*}\right)\right]$.

A sequence $\left\{\Omega_{n}(\alpha)\right.$ \} can be constructed as in Lemma 3 so that

$$
\lim _{n \rightarrow \infty} G_{i}\left(\Omega_{n}(\alpha)\right)=G_{i}\left(\Omega^{*}\right)+\alpha\left\langle D G_{\Omega^{*}}^{l}, \chi_{\Omega}-\chi_{\Omega^{*}}\right\rangle+o(\alpha), \quad i=1, \ldots, m .
$$

If $i \in I\left(\Omega^{*}\right)$ then $G_{l}\left(\Omega^{*}\right)=0$. By the definition of $o(\alpha)$, there exists $\delta^{\prime}>0$ such that $|o(\alpha)|<\frac{1}{2}\left|\left\langle D G_{\Omega^{*}}^{i}, \chi_{\Omega}-\chi_{\Omega^{*}}\right\rangle\right| \alpha$ for $\alpha<\delta^{\prime}$. Therefore from (3), (5) becomes

$$
\lim _{n \rightarrow \infty} G_{i}\left(\Omega_{n}(\alpha)\right)<\frac{\alpha}{2}\left\langle D G_{\Omega^{*}}^{t}, \chi_{\Omega}-\chi_{\Omega^{*}}\right\rangle<0 \text { for } \alpha<\delta^{\prime}
$$

and hence, for any $\alpha<\delta^{\prime}$ there exists $M_{\alpha}>0$ such that $G_{t}\left(\Omega_{n}(\alpha)\right)<0$ for all $n>M_{\alpha}$.

If $i \notin I\left(\Omega^{*}\right)$ then $G_{i}\left(\Omega^{*}\right)<0$, and (5) becomes $\lim _{n \rightarrow \infty}\left(\Omega_{n}(\alpha)\right) \rightarrow G_{i}\left(\Omega^{*}\right)<0$ as $\alpha \rightarrow 0$. Therefore, there exists $\delta^{\prime \prime}>0$ so that for any $\alpha<\delta^{\prime \prime}$ there exists $M_{\alpha}>0$ such that $G_{i}\left(\Omega_{n}(\alpha)\right)<0$ for all $n>M_{\alpha}$.

We have shown that there exists $\delta=\min \left(\delta^{\prime}, \delta^{\prime \prime}\right)>0$, such that for any $\alpha<\delta$ there is $N_{\alpha}>0$ so that $G_{t}\left(\Omega_{n}(\alpha)\right)<0$, for all $n>N_{\alpha}, i=1, \ldots, m$. Since $\Omega^{*}$ is a local minimum we have, for any $\alpha<\delta$,

$$
F\left(\Omega_{n}(\alpha)\right) \geqslant F\left(\Omega^{*}\right) \text { for all } n>N_{\alpha} .
$$

Therefore

$$
\lim _{n \rightarrow \infty} F\left(\Omega_{n}(\alpha)\right) \geqslant F\left(\Omega^{*}\right) \text { for } \alpha<\delta
$$

Applying the sequence $\left\{\Omega_{n}(\alpha)\right\}$ to (4), we obtain

$$
\lim _{n \rightarrow \infty} F\left(\Omega_{n}(\alpha)\right)=F\left(\Omega^{*}\right)+\alpha^{2}\left\langle D^{2} L_{\Omega^{*}},\left(\chi_{\Omega}-\chi_{\Omega^{*}}\right)^{2}\right\rangle+o\left(\alpha^{2}\right) .
$$

Dividing both sides by $\alpha^{2}$ and letting $\alpha \rightarrow 0$ we have

$$
\left\langle D^{2} L_{\Omega^{*}},\left(\chi_{\Omega}-\chi_{\Omega^{*}}\right)^{2}\right\rangle \geqslant 0
$$

for all $\Omega \in S$ satisfying (3). Q.E.D. 
The following theorem gives first-order sufficient conditions for optimality. The theorem follows the spirit of Theorems 5.3 and 5.6 in [10] and can be proved by a similar argument.

Theorem 4 (First-Order Sufficient Condition). Suppose $\Omega^{*} \in S$ and suppose $L(\Omega)=F(\Omega)+\sum_{i=1}^{m} \lambda_{i}(\Omega)$ is a Lagrangian function for problem (1) at $\Omega^{*}$. If there is $\gamma>0$ such that

$$
\left\langle D L_{\Omega^{*}}, \chi_{\Omega}-\chi_{\Omega^{*}}\right\rangle \geqslant \gamma \cdot \rho\left(\Omega, \Omega^{*}\right) \text { for all } \Omega \in S
$$

then there exist $\alpha>0$ and $\beta>0$ such that

$$
F(\Omega) \geqslant F\left(\Omega^{*}\right)+\alpha \cdot \rho\left(\Omega, \Omega^{*}\right) \text { for all } \Omega \in S
$$

with $\rho\left(\Omega, \Omega^{*}\right) \leqslant \beta$.

If we relax the first-order sufficient condition in the above theorem then we need to impose a second-order condition on the set $\Omega$ for which the first-order condition is violated, that is,

$$
\left\langle D L_{\Omega^{*}}, \chi_{\Omega}-\chi_{\Omega^{*}}\right\rangle\left\langle\gamma \cdot \rho\left(\Omega, \Omega^{*}\right) .\right.
$$

Theorem 5 (Second-Order Sufficient Condition). Suppose

(i) $\Omega^{*} \in S$,

(ii) $L(\Omega)=F(\Omega)+\sum_{i=1}^{m} \lambda_{i} G_{i}(\Omega)$ is a Lagrangian function for problem (1),

(iii) $L$ is twice differentiable at $\Omega_{0}$, and

(iv) there exists $\gamma>0$ such that $\left\langle D^{2} L_{\Omega^{*}},\left(\chi_{\Omega}-\chi_{\Omega^{*}}\right)^{2}\right\rangle \geqslant \gamma \rho^{2}\left(\Omega, \Omega^{*}\right)$ in $a$ neighborhood of $\Omega^{*}$ in $S$. Then $\Omega^{*}$ is a local minimum of $F$ in $S$.

Proof. The proof is straightforward by using Lemma 5. Q.E.D.

\section{Acknowledgment}

The authors greatly appreciate helpful comments and suggestions received from the referee which led to a considerable improvement of the original version of this paper.

\section{References}

[1] D. Begis and R. Glowinski, “Application de la méthode des éléments finis à l'approximation d'un problème de domaine optimal. Méthodes de résolution des problèmes approchés", Appl. Math. Optim. 2 (1975), 130-169. 
[2] A. Ben-Tal and J. Zowe, "A unified theory of first and second order conditions for extremum problems in topoological vector spaces", in Mathemattcal programming study 19 (ed. M. Guignard), (North-Holland, Amsterdam, 1981).

[3] J. Cea, A. Gioan and J. Michel, "Queques résultats sur l'identification de domaines", Calcolo 10 (1973), 133-145.

[4] M. R. Hestenes, Optimization theory - The fintte-dimenstonal case (Wiley, New York, 1975).

[5] A.D. Ioffe, "Necessary and sufficient conditions for a local minimum. 3: Second order conditions and augmented duality", SIAM J. Control Optim. 17 (1979), 266-288.

[6] H. L. Lai and S. S. Yang, "Saddle point and duality in the optimization theory of convex set functions", J. A ustral. Math. Soc. Ser. B 24 (1982), 130-137.

[7] H. L. Lai, S. S. Yang and G. R. Hwang, "Duality in mathematical programming of set functions: On Fenchel duality theorem", J. Math. Anal. Appl. 95 (1983), 223-234.

[8] D. G. Luenberger, Optimization by vector space methods (Wiley, New York, 1969).

[9] O. L. Mangasarian, Nonlinear programming (McGraw Hill, New York, 1969).

[10] H. Maurer and J. Zowe, "First and second-order necessary and sufficient optimality conditions for infinite-dimensional programming problems", Math. Programming 16 (1979), 98-110.

[11] Robert J. T. Morris, "Optimal constrained selection of a measurable subset", J. Math. Anal. Appl. 70 (1979), 546-562.

[12] P. K. C. Wang, "On a class of optimization problems involving domain variations", in International symposium on new trends in system analysis, Versailles, France, (Dec. 1976), Lecture Notes in Control and Information Sciences 2 (Springer-Verlag, Berlin, 1977), 49. 